\title{
Product Collaborative Innovation of Project-based Supply Chain under the Influence of Knowledge Input
}

\author{
Wei Chen ${ }^{1,2}$, Yifei $\mathrm{Hao}^{3 *}$, Niqianjun $\mathrm{Jin}^{4}$ \\ ${ }^{1}$ Research Center for Enterprise Management, Chongqing Technology and Business University, Chongqing 400067, China \\ ${ }^{2}$ School of Management, Chongqing Technology and Business University, Chongqing 400067, China \\ ${ }^{3}$ College of Mathematics and Statistics, Chongqing Technology and Business University, Chongqing 400067, China \\ ${ }^{4}$ CCTEG Chongqing Research Institute, Chongqing 400039, China
}

Corresponding Author Email: haoyf@ctbu.edu.cn

https://doi.org/10.18280/jesa.520304

Received: 19 February 2019

Accepted: 15 May 2019

\section{Keywords:}

project-based supply chain, collaborative innovation, knowledge input, differential game

\begin{abstract}
In a project-based supply chain, the knowledge input of member enterprises directly bears on the success of the collaborative innovation. Considering the effect of enterprise knowledge input on product collaborative innovation, this paper applies the differential game theory to coordinate the product collaborative innovation in a three-stage supply chain, consisting of a manufacturer, a supplier and a seller. The equilibrium outcomes of centralized decisionmaking mode and two decentralized decision-making modes, namely, the Stackelberg masterslave game and the Nash non-cooperative game, were subjected to a comparative analysis. The results show that, under the centralized decision-making mode, the knowledge input and collaborative innovation of enterprises were optimized, leading to the maximal overall revenue of the supply chain. Under the two decentralized decision-making modes, the manufacturer had the same knowledge input; however, the supplier and the seller both had higher knowledge input amount, enterprise revenue and collaborative innovation level under the Stackelberg master-slave game than under the Nash non-cooperative game; the increase ratios of knowledge input of the supplier and the seller are equal to the proportions of their respective knowledge input costs that are shared.
\end{abstract}

\section{INTRODUCTION}

The project-based supply chain mainly focuses on the project innovation, it's a cross-organizational structure mode with specific functions, and it is formed by upstream and downstream enterprises in the supply chain with heterogeneous and complementary knowledge resources in order to achieve a common goal $[1,2]$. Compared with the traditional supply chain, the project-based supply chain emphasizes more on the collaborative innovation among upstream and downstream enterprises based on projects, so it perfectly fits the innovation needs of project-based enterprises $[3,4]$. Under the strategic background of national innovationdriven development, the collaborative innovation of projectbased supply chain has gradually become the focus of attention in the theoretical and practical research fields.

At present, domestic and foreign researches on the collaborative innovation of project-based supply chain mainly focus on the following two aspects: the first is to study the problems of collaborative innovation of project-based supply chain from the perspective of knowledge management, which mainly studies the influence of cross-organizational knowledge flow in the project-based supply chain on the collaborative innovation projects (project value increment, innovation performance or cooperative performance) from theoretical and empirical perspectives, such as $\mathrm{Wu}$ [5] considered the effort cost relationship between the control objectives of the cross-enterprise knowledge flow in the supply chain, and his research had built and analyzed a knowledge flow-based project-oriented supply chain crossenterprise cooperation incentive model; Saunders et al. [6] used multi-case analysis to analyze the influence of suppliers' early input of knowledge resources in the project collaborative innovation on the cooperation performance in the projectbased supply chain; Nasr et al. [7] used cases to study the influence of knowledge exchange on the cross-enterprise collaborative innovation projects in the supply chain. The second aspect is to study the problems of project-based supply chain collaborative innovation from the perspective of incentives, which mainly considers the factors such as the efforts of partnership, the preference of fairness, payment cost, distribution coefficient and reciprocal preference, etc.; through mathematical modeling, these factors' influence on the project-based supply chain collaborative innovation was analyzed, and then corresponding incentive measures have been proposed according to the research results, for instance, Lai et al. [8] established a dynamic reputation incentive model to study the influence of implicit reputation factor on the value increment of cross-enterprise collaborate innovation in the project-based supply chain; $\mathrm{Wu}$ and Tang [9] applied the principal-agent theory to establish a cross-organizational collaborative innovation incentive model based on monitoring signals in the project-based supply chain, and analyzd the influence of reward and punishment mechanism on crossorganizational collaborative innovation; Preeker and Giovanni [10] analyzed the incentive effect of various types of contracts (price discounts, repurchase and cost sharing contracts, etc.) on the member companies in different stages of the project 
collaborative innovation based on the motivation (a comparative case study of five high-tech companies in the Netherlands had been conducted) of member companies carrying supply chain in the supply chain.

Considering that knowledge is the core element of innovation, to some extent, the project-based supply chain collaborative innovation activities can be regarded as an infinite cyclic process of knowledge input, knowledge sharing and knowledge innovation [11, 12], during which the knowledge input of member companies is the key factor affecting the success of collaborative innovation projects [13], therefore, stimulating the knowledge input of member companies is the primary problem for improving the collaborative innovation behaviors in the project-based supply chain [14]. Although previous researches have carried out related studies on the collaborative innovation of project-based supply chain from the perspective of knowledge management and incentives, there is still a lack of research on the collaborative innovation of project-based supply chain considering the knowledge input of member companies. In addition, since the project-based supply chain crossorganizational collaborative innovation activities usually have the characteristics of long-term, complex and dynamic, they are a continuous dynamic process, with market as the orientation [15]. In contrast, the previous two-stage supply chain with the manufacturer and the core component supplier as the main body of collaborative innovation can no longer accord with the reality of project-based supply chain, therefore, for the cross-enterprise collaborative innovation main bodies in the project-based supply chain, besides the manufacturer and the key component supplier, it is also necessary to introduce sellers who directly face the consumer market and understand the product market knowledge. In view of this, this paper takes the product collaborative innovation projects of the project-based supply chain as the research object, comprehensively considers the influence of member enterprises' knowledge input and time effect, and constructs a differential game model for the three-stage project-based supply chain collaborative innovation, it studies the optimal knowledge input, optimal revenue, optimal innovation level, and overall supply chain revenue status of the manufacturer, the supplier, and the seller under different game scenarios, and analyzes the coordination mechanism of the collaborative innovation behaviors in the project-based supply chain, in the hopes of providing some theoretical guidance for the projectbased supply chain to carry out collaborative innovation activities.

\section{PROBLEM DESCRIPTION AND RESEARCH HYPOTHESIS}

In order to quantitatively study the knowledge input decision-making problem of project-based supply chain collaborative innovation, this paper constructs a three-stage project-based supply chain system consisted of a single product manufacturer, a single component supplier and a single product seller (as shown in Figure 1), the three parties respectively invest in product technical knowledge, component technical knowledge and product market knowledge to carry out product collaborative innovation projects. To conduct the above-mentioned research, the following hypotheses are made:

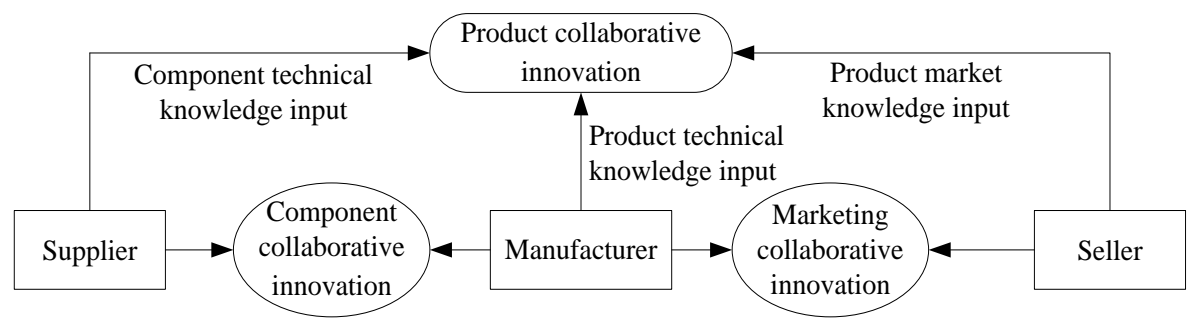

Figure 1. Framework of project-based supply chain collaborative innovation

Hypothesis 1: The knowledge input amounts of the manufacturer, the supplier, and the seller at moment $t$ are $k_{m}(t)$, $k_{s}(t)$ and $k_{r}(t)$, respectively, where, $t \in[0,+\infty)$ is the time variable.

Hypothesis 2: The product innovation level $N(t)$ depends on the knowledge input amounts of the manufacturer, the supplier, and the seller, it is a strictly downward convex function, and its dynamic process is as follows:

$$
\left\{\begin{array}{l}
\frac{\mathrm{d} N(t)}{\mathrm{d} t}=\lambda_{m} k_{m}(t)+\lambda_{s} k_{s}(t)+\lambda_{r} k_{r}(t)-\delta N(t) \\
N(0)=N_{0} \geq 0
\end{array}\right.
$$

where, $\lambda_{m}, \lambda_{s}$ and $\lambda_{r}$ respectively represent the influence of the knowledge input of the manufacturer, the supplier and the seller on the product innovation, namely the coefficients of innovation influence; $\delta$ represents the relative attenuation rate of innovation level caused by outdated knowledge and concept, behindhand technology, and other reasons, namely the innovation attenuation coefficient; $N_{0}$ represents the product initial innovation level.

Hypothesis 3: Referring to the cost function in Literature [16], we assume that the knowledge input costs of manufacturer, supplier and seller are quadratic functions of knowledge inputs, that is:

$$
C_{m}=\frac{\mu_{m}}{2} k_{m}^{2}(t), C_{s}=\frac{\mu_{s}}{2} k_{s}^{2}(t), C_{r}=\frac{\mu_{r}}{2} k_{r}^{2}(t),
$$

where, $\mu_{m}, \mu_{s}$ and $\mu_{r}$ represent the knowledge input cost coefficients of the manufacturer, the supplier and the seller, the upward convex characteristic of knowledge input cost reflects the enterprise marginal cost increase principle, that is, the greater the knowledge input of member companies, the greater the cost of further investment.

Hypothesis 4: The overall revenue brought by a product collaborative innovation project to the supply chain is:

$$
\pi(t)=\varphi+\eta N(t)
$$


where, $\eta$ represents the degree of influence of the product innovation level on the overall revenue of the supply chain, $\varphi$ is a constant.

Hypothesis 5: The manufacturer, the supplier, and the seller are rational decision makers who seek to maximize their own profits, and the three parties have complete information and the same discount rate $r(r>0)$; the cooperative three parties divide the total revenue brought by the product collaborative innovation projects according to an agreed ratio, the supplier obtains $\alpha_{1}\left(0<\alpha_{1}<1-\alpha_{2}\right)$, the seller obtains $\alpha_{2}$ $\left(0<\alpha_{2}<1-\alpha_{1}\right)$, and the manufacturer obtains $1-\alpha_{1}-\alpha_{2}$; moreover, the cost-sharing ratios of the knowledge input by the manufacturer to the supplier and the seller are $\beta_{1}(t)$ and $\beta_{2}(t)$, respectively.

In addition, for the parameters mentioned in the above hypotheses, we assume that they are normal numbers that are independent of time.

\section{DECENTRALIZED DECISION-MAKING MODE}

\subsection{Stackelberg master-slave game}

Assume that in the project-based supply chain, the manufacturer is the core enterprise, and is the leader of the product collaborative innovation project. The supplier and the seller are followers, and each member company takes the maximization of their respective revenue as the decisionmaking goal. The decision-making problem is based on the master-slave relationship. First, the manufacturer determines the optimal knowledge input amount $k_{m}$ of its own, and its cost-sharing ratios of the knowledge input to the supplier and the seller are $\beta_{1}$ and $\beta_{2}$; after the supplier and the seller have observed the decision of the manufacturer, they respectively select their own optimal knowledge input amounts $k_{s}$ and $k_{r}$. Then the decision-making questions for the manufacturer, the supplier and the seller are:

$$
\begin{aligned}
& J_{m}=\max _{k_{m}} \int_{0}^{\infty} e^{-r t}\left[\left(1-\alpha_{1}-\alpha_{2}\right)(\varphi+\eta N(t))-\frac{\mu_{m}}{2} k_{m}^{2}-\beta_{1} \frac{\mu_{s}}{2} k_{s}^{2}-\beta_{2} \frac{\mu_{r}}{2} k_{r}^{2}\right] \mathrm{d} t \\
& J_{s}=\max _{k_{s}} \int_{0}^{\infty} e^{-r t}\left[\alpha_{1}(\varphi+\eta N(t))-\left(1-\beta_{1}\right) \frac{\mu_{s}}{2} k_{s}^{2}\right] \mathrm{d} t \\
& J_{r}=\max _{k_{r}} \int_{0}^{\infty} e^{-r t}\left[\alpha_{2}(\varphi+\eta N(t))-\left(1-\beta_{2}\right) \frac{\mu_{r}}{2} k_{r}^{2}\right] \mathrm{d} t
\end{aligned}
$$

$$
\begin{aligned}
& k_{m}^{*}=\frac{\lambda_{m} \eta\left(1-\alpha_{1}-\alpha_{2}\right)}{\mu_{m}(r+\delta)} \\
& k_{s}^{*}=\frac{\lambda_{s} \eta\left(2-\alpha_{1}-2 \alpha_{2}\right)}{2 \mu_{s}(r+\delta)} \\
& r V_{m}(N)=\max _{k_{m}}\left[\left(1-\alpha_{1}-\alpha_{2}\right)(\varphi+\eta N)-\frac{\mu_{m}}{2} k_{m}^{2}-\beta_{1} \frac{\mu_{s}}{2} k_{s}^{2}-\beta_{2} \frac{\mu_{r}}{2} k_{r}^{2}+V_{m}^{\prime}(N)\left(\lambda_{m} k_{m}+\lambda_{s} k_{s}+\lambda_{r} k_{r}-\delta N\right)\right] \\
& r V_{s}(N)=\max _{k_{s}}\left[\alpha_{1}(\varphi+\eta N)-\left(1-\beta_{1}\right) \frac{\mu_{s}}{2} k_{s}^{2}+V_{s}^{\prime}(N)\left(\lambda_{m} k_{m}+\lambda_{s} k_{s}+\lambda_{r} k_{r}-\delta N\right)\right] \\
& r V_{r}(N)=\max _{k_{r}}\left[\alpha_{2}(\varphi+\eta N)-\left(1-\beta_{2}\right) \frac{\mu_{r}}{2} k_{r}^{2}+V_{r}^{\prime}(N)\left(\lambda_{m} k_{m}+\lambda_{s} k_{s}+\lambda_{r} k_{r}-\delta N\right)\right]
\end{aligned}
$$

Prove: formulas (3), (4) and (5) were respectively subject to the dynamic programming method, and the Hamilton-Jacobi-

where, $V_{i}=e^{r t} J_{i}(i=m, s, r)$ is the optimal innovation revenue function of the member companies in the supply chain. Using the inverse induction method, first of all, respectively let the HJB equations of the supplier and the seller have a firstorder partial derivative of $k_{s}$ and $k_{r}$ equal to zero, then the optimal knowledge input amounts of the supplier and the seller are:

$$
k_{s}^{*}=\frac{\lambda_{s} V_{s}^{\prime}}{\mu_{s}\left(1-\beta_{1}\right)}
$$

$$
k_{r}^{*}=\frac{\lambda_{r} V_{r}^{\prime}}{\mu_{r}\left(1-\beta_{2}\right)}
$$

Since a rational manufacturer would determine its optimal strategy according to the decisions of the supplier and the seller, by substituting formulas (14) and (15) into formula (11), we can obtain the optimal knowledge input and the optimal cost-sharing ratios as follows:

$$
k_{m}^{*}=\frac{\lambda_{m} V_{m}^{\prime}}{\mu_{m}}
$$




$$
\beta_{1}^{*}=\frac{2 V_{m}{ }^{\prime}-V_{s}^{\prime}}{2 V_{m}{ }^{\prime}+V_{s}^{\prime}}
$$

$$
\beta_{2}^{*}=\frac{2 V_{m}^{\prime}-V_{r}^{\prime}}{2 V_{m}^{\prime}+V_{r}^{\prime}}
$$

Substituting formulas (14)-(18) into the right end of the HJB equation, respectively, then we can get:

$$
\begin{gathered}
r V_{m}(N)=\left[\left(1-\alpha_{1}-\alpha_{2}\right) \eta-\delta V_{m}^{\prime}(N)\right] N+\left(1-\alpha_{1}-\alpha_{2}\right) \varphi+\frac{\lambda_{m}^{2}\left(V_{m}^{\prime}(N)\right)^{2}}{2 \mu_{m}}+\frac{\lambda_{s}^{2}\left[2 V_{m}^{\prime}(N)+V_{s}^{\prime}(N)\right]^{2}}{8 \mu_{s}}+\frac{\lambda_{r}^{2}\left[2 V_{m}^{\prime}(N)+V_{r}^{\prime}(N)\right]^{2}}{8 \mu_{r}} \\
r V_{s}(N)=\left[\alpha_{1} \eta-\delta V_{s}^{\prime}(N)\right] N+\alpha_{1} \varphi+\frac{\lambda_{m}^{2} V_{s}^{\prime}(N) V_{m}^{\prime}(N)}{\mu_{m}}+\frac{\lambda_{s}^{2} V_{s}^{\prime}(N)\left[2 V_{m}^{\prime}(N)+V_{s}^{\prime}(N)\right]}{4 \mu_{s}}+\frac{\lambda_{r}^{2} V_{s}^{\prime}(N)\left[2 V_{m}^{\prime}(N)+V_{r}^{\prime}(N)\right]}{2 \mu_{r}} \\
r V_{r}(N)=\left[\alpha_{2} \eta-\delta V_{r}^{\prime}(N)\right] N+\alpha_{2} \varphi+\frac{\lambda_{m}^{2} V_{r}^{\prime}(N) V_{m}^{\prime}(N)}{\mu_{m}}+\frac{\lambda_{r}^{2} V_{r}^{\prime}(N)\left[2 V_{m}^{\prime}(N)+V_{r}^{\prime}(N)\right]}{4 \mu_{r}}+\frac{\lambda_{s}^{2} V_{r}^{\prime}(N)\left[2 V_{m}^{\prime}(N)+V_{s}^{\prime}(N)\right]}{2 \mu_{s}}(2)
\end{gathered}
$$

From the form of formulas (19)-(21) we can know that, the optimal innovation revenue functions $V_{m}(N), V_{s}(N)$ and $V_{r}(N)$ should be linear functions. Assume there are $V_{m}(N)=f_{1} N+f_{2}, V_{s}(N)=g_{1} N+g_{2}$, and $V_{r}(N)=h_{1} N+h_{2}$, where, $f_{1}, f_{2}, g_{1}, g_{2}, h_{1}, h_{2}$ are undetermined constants, then $V_{m}(N), V_{s}(N), V_{r}(N)$ and their derivatives were substituted into formulas (19)-(21) and sorted out to get :

$$
\begin{gathered}
r\left(f_{1} N+f_{2}\right)=\left[\left(1-\alpha_{1}-\alpha_{2}\right) \eta-\delta f_{1}\right] N+\left(1-\alpha_{1}-\alpha_{2}\right) \varphi+\frac{\lambda_{m}^{2} f_{1}^{2}}{2 \mu_{m}}+\frac{\lambda_{s}^{2}\left[2 f_{1}+g_{1}\right]^{2}}{8 \mu_{s}}+\frac{\lambda_{r}^{2}\left[2 f_{1}+h_{1}\right]^{2}}{8 \mu_{r}} \\
r\left(g_{1} N+g_{2}\right)=\left(\alpha_{1} \eta-\delta g_{1}\right) N+\alpha_{1} \varphi+\frac{\lambda_{m}^{2} f_{1} g_{1}}{\mu_{m}}+\frac{\lambda_{s}^{2} g_{1}\left[2 f_{1}+g_{1}\right]}{4 \mu_{s}}+\frac{\lambda_{r}^{2} g_{1}\left[2 f_{1}+h_{1}\right]}{2 \mu_{r}} \\
r\left(h_{1} N+h_{2}\right)=\left(\alpha_{2} \eta-\delta h_{1}\right) N+\alpha_{2} \varphi+\frac{\lambda_{m}^{2} f_{1} h_{1}}{\mu_{m}}+\frac{\lambda_{r}^{2} h_{1}\left[2 f_{1}+h_{1}\right]}{4 \mu_{r}}+\frac{\lambda_{s}^{2} h_{1}\left[2 f_{1}+g_{1}\right]}{2 \mu_{s}}
\end{gathered}
$$

Respectively compare the coefficient terms of $N$ and the constant terms on the left and right sides of formulas (22), (23), and (24), and then obtain the parameters as:

$$
\begin{gathered}
f_{1}=\frac{\left(1-\alpha_{1}-\alpha_{2}\right) \eta}{r+\delta}, f_{2}=\frac{\left(1-\alpha_{1}-\alpha_{2}\right) \varphi}{r}+\frac{\lambda_{m}^{2}\left(1-\alpha_{1}-\alpha_{2}\right)^{2} \eta^{2}}{2 r \mu_{m}(r+\delta)^{2}}+\frac{\lambda_{s}^{2}\left(2-\alpha_{1}-2 \alpha_{2}\right)^{2} \eta^{2}}{8 r \mu_{s}(r+\delta)^{2}}+\frac{\lambda_{r}^{2}\left(2-2 \alpha_{1}-\alpha_{2}\right)^{2} \eta^{2}}{8 r \mu_{r}(r+\delta)^{2}} \\
g_{1}=\frac{\alpha_{1} \eta}{r+\delta}, g_{2}=\frac{\alpha_{1} \varphi}{r}+\frac{\lambda_{m}^{2} \eta^{2} \alpha_{1}\left(1-\alpha_{1}-\alpha_{2}\right)}{r \mu_{m}(r+\delta)^{2}}+\frac{\lambda_{s}^{2} \eta^{2} \alpha_{1}\left(2-\alpha_{1}-2 \alpha_{2}\right)}{4 r \mu_{s}(r+\delta)^{2}}+\frac{\lambda_{r}^{2} \eta^{2} \alpha_{1}\left(2-2 \alpha_{1}-\alpha_{2}\right)}{2 r \mu_{r}(r+\delta)^{2}} \\
h_{1}=\frac{\alpha_{2} \eta}{r+\delta}, h_{2}=\frac{\alpha_{2} \varphi}{r}+\frac{\lambda_{m}^{2} \eta^{2} \alpha_{2}\left(1-\alpha_{1}-\alpha_{2}\right)}{r \mu_{m}(r+\delta)^{2}}+\frac{\lambda_{r}^{2} \eta^{2} \alpha_{2}\left(2-2 \alpha_{1}-\alpha_{2}\right)}{4 r \mu_{r}(r+\delta)^{2}}+\frac{\lambda_{s}^{2} \eta^{2} \alpha_{2}\left(2-\alpha_{1}-2 \alpha_{2}\right)}{2 r \mu_{s}(r+\delta)^{2}}
\end{gathered}
$$

By substituting $V_{m}^{\prime}(N)=f_{1}, V_{s}^{\prime}(N)=g_{1}$ and $V_{r}^{\prime}(N)=h_{1}$ into formulas (14)-(18), we can prove the proposition 1 . Wherein, from $0 \leq \beta_{1} \leq 1$ and $0<\alpha_{1}<1-\alpha_{2}$ we can get $0<$ $\alpha_{1} \leq \frac{2}{3}\left(1-\alpha_{2}\right)$; similarly, we can also get $0<\alpha_{2} \leq \frac{2}{3}(1-$ $\left.\alpha_{1}\right)$.

$$
\begin{gathered}
V_{m}^{*}\left(N^{*}\right)=\frac{\eta\left(1-\alpha_{1}-\alpha_{2}\right)}{r+\delta} N^{*}+\frac{\varphi\left(1-\alpha_{1}-\alpha_{2}\right)}{r}+\frac{\lambda_{m}^{2} \eta^{2}\left(1-\alpha_{1}-\alpha_{2}\right)^{2}}{2 r \mu_{m}(r+\delta)^{2}}+\frac{\lambda_{s}^{2} \eta^{2}\left(2-\alpha_{1}-2 \alpha_{2}\right)^{2}}{8 r \mu_{s}(r+\delta)^{2}}+\frac{\lambda_{r}^{2} \eta^{2}\left(2-2 \alpha_{1}-\alpha_{2}\right)^{2}}{8 r \mu_{r}(r+\delta)^{2}} \\
V_{s}^{*}\left(N^{*}\right)=\frac{\eta \alpha_{1}}{r+\delta} N^{*}+\frac{\varphi \alpha_{1}}{r}+\frac{\lambda_{m}^{2} \eta^{2} \alpha_{1}\left(1-\alpha_{1}-\alpha_{2}\right)}{r \mu_{m}(r+\delta)^{2}}+\frac{\lambda_{s}^{2} \eta^{2} \alpha_{1}\left(2-\alpha_{1}-2 \alpha_{2}\right)}{4 r \mu_{s}(r+\delta)^{2}}+\frac{\lambda_{r}^{2} \eta^{2} \alpha_{1}\left(2-2 \alpha_{1}-\alpha_{2}\right)}{2 r \mu_{r}(r+\delta)^{2}}
\end{gathered}
$$




$$
\begin{gathered}
V_{r}^{*}\left(N^{*}\right)=\frac{\eta \alpha_{2}}{r+\delta} N^{*}+\frac{\varphi \alpha_{2}}{r}+\frac{\lambda_{m}^{2} \eta^{2} \alpha_{2}\left(1-\alpha_{1}-\alpha_{2}\right)}{r \mu_{m}(r+\delta)^{2}}+\frac{\lambda_{r}^{2} \eta^{2} \alpha_{2}\left(2-2 \alpha_{1}-\alpha_{2}\right)}{4 r \mu_{r}(r+\delta)^{2}}+\frac{\lambda_{s}^{2} \eta^{2} \alpha_{2}\left(2-\alpha_{1}-2 \alpha_{2}\right)}{2 r \mu_{s}(r+\delta)^{2}} \\
V^{*}\left(N^{*}\right)=\frac{\eta}{r+\delta} N^{*}+\frac{\varphi}{r}+\frac{\lambda_{m}^{2} \eta^{2}\left(1-\alpha_{1}-\alpha_{2}\right)\left(1+\alpha_{1}+\alpha_{2}\right)}{2 r \mu_{m}(r+\delta)^{2}}+\frac{\lambda_{s}^{2} \eta^{2}\left(2-\alpha_{1}-2 \alpha_{2}\right)\left(2+\alpha_{1}+2 \alpha_{2}\right)}{8 r \mu_{s}(r+\delta)^{2}}+\frac{\lambda_{r}^{2} \eta^{2}\left(2-2 \alpha_{1}-\alpha_{2}\right)\left(2+2 \alpha_{1}+\alpha_{2}\right)}{8 r \mu_{r}(r+\delta)^{2}}
\end{gathered}
$$

where, the optimal innovation level is

$$
N^{*}(t)=\frac{\lambda_{m} k_{m}^{*}+\lambda_{s} k_{s}^{*}+\lambda_{r} k_{r}^{*}}{\delta}+\left(N_{0}-\frac{\lambda_{m} k_{m}^{*}+\lambda_{s} k_{s}^{*}+\lambda_{r} k_{r}^{*}}{\delta}\right) e^{-\delta t} .
$$

Prove: by substituting formulas (14), (15) and (16) into formula (1) we can get:

$$
N^{\prime}(t)=\left(\lambda_{m} k_{m}^{*}+\lambda_{s} k_{s}^{*}+\lambda_{r} k_{r}^{*}\right)-\delta N(t)
$$

From the general solution formula of the first-order linear ordinary differential equation, we can find the special solution that satisfies $N(0)=N_{0}$ as follows:

$$
\begin{aligned}
& N^{*}(t)=\frac{\lambda_{M} k_{M}^{*}+\lambda_{S} k_{S}^{*}+\lambda_{R} k_{R}^{*}}{\delta} \\
& +\left(N_{0}-\frac{\lambda_{M} k_{M}^{*}+\lambda_{S} k_{S}^{*}+\lambda_{R} k_{R}^{*}}{\delta}\right) e^{-\delta t}
\end{aligned}
$$

By respectively substituting $N^{*}(t)$ and the values of $f_{1}$, $f_{2}, g_{1}, g_{2}, h_{1}, h_{2}$ into the optimal innovation revenue functions of the manufacture, the supplier, the seller and the supply chain, proposition 2 can be proved.

\subsection{Nash non-cooperative game}

Assume that in a project-based supply chain, the three parties involved in the product collaborative innovation project are equal, their decisions are independent, and they take actions at the same time so as to maximize their own profits. At this point, a rational decision maker will not share the cost of knowledge input for other companies. Therefore, there is $\beta_{1}(t)=\beta_{2}(t)=0, t \in[0,+\infty)$, and the objective functions of the manufacturer, the supplier, and the seller are:

$$
\begin{gathered}
J_{m}=\max _{k_{m}} \int_{0}^{\infty} e^{-r t}\left[\left(1-\alpha_{1}-\alpha_{2}\right)(\varphi+\eta N(t))-\frac{\mu_{m}}{2} k_{m}^{2} \mathrm{~d} t\right. \\
J_{s}=\max _{k_{s}} \int_{0}^{\infty} e^{-r t}\left[\alpha_{1}(\varphi+\eta N(t))-\frac{\mu_{s}}{2} k_{s}^{2}\right] \mathrm{d} t \\
J_{r}=\max _{k_{r}} \int_{0}^{\infty} e^{-r t}\left[\alpha_{2}(\varphi+\eta N(t))-\frac{\mu_{r}}{2} k_{r}^{2}\right] \mathrm{d} t
\end{gathered}
$$

Proposition 3. In the case of Nash non-cooperative game, the optimal knowledge input amounts of the manufacturer, the supplier, and the seller in the three-stage project-based supply chain are:

$$
\begin{gathered}
k_{m}^{* * *}=\frac{\lambda_{m} \eta\left(1-\alpha_{1}-\alpha_{2}\right)}{\mu_{m}(r+\delta)} \\
k_{s}^{* * *}=\frac{\lambda_{s} \eta \alpha_{1}}{\mu_{s}(r+\delta)} \\
k_{r}^{* * *}=\frac{\lambda_{r} \eta \alpha_{2}}{\mu_{r}(r+\delta)}
\end{gathered}
$$

Prove: same as the proof of proposition 1, omitted.

Proposition 4. In the case of Nash non-cooperative game, the optimal innovation revenues of the manufacturer, the supplier, the seller, and the supply chain in the three-stage project-based supply chain are:

$$
\begin{gathered}
V_{m}^{* * *}\left(N^{* * *}\right)=\frac{\eta\left(1-\alpha_{1}-\alpha_{2}\right)}{r+\delta} N^{* * *}+\frac{\varphi\left(1-\alpha_{1}-\alpha_{2}\right)}{r}+\frac{\lambda_{m}{ }^{2} \eta^{2}\left(1-\alpha_{1}-\alpha_{2}\right)^{2}}{2 r \mu_{m}(r+\delta)^{2}}+\frac{\lambda_{s}{ }^{2} \eta^{2} \alpha_{1}\left(1-\alpha_{1}-\alpha_{2}\right)}{r \mu_{s}(r+\delta)^{2}}+\frac{\lambda_{r}{ }^{2} \eta^{2} \alpha_{2}\left(1-\alpha_{1}-\alpha_{2}\right)}{r \mu_{r}(r+\delta)^{2}} \\
V_{s}^{* * *}\left(N^{* * *}\right)=\frac{\eta \alpha_{1}}{r+\delta} N^{* * *}+\frac{\varphi \alpha_{1}}{r}+\frac{\lambda_{m}{ }^{2} \eta^{2} \alpha_{1}\left(1-\alpha_{1}-\alpha_{2}\right)}{r \mu_{m}(r+\delta)^{2}}+\frac{\lambda_{s}{ }^{2} \eta^{2} \alpha_{1}{ }^{2}}{2 r \mu_{s}(r+\delta)^{2}}+\frac{\lambda_{r}{ }^{2} \eta^{2} \alpha_{1} \alpha_{2}}{r \mu_{r}(r+\delta)^{2}} \\
V_{r}^{* * *}\left(N^{* * *}\right)=\frac{\eta \alpha_{2}}{r+\delta} N^{* * *}+\frac{\varphi \alpha_{2}}{r}+\frac{\lambda_{m}{ }^{2} \eta^{2} \alpha_{2}\left(1-\alpha_{1}-\alpha_{2}\right)}{r \mu_{m}(r+\delta)^{2}}+\frac{\lambda_{r}{ }^{2} \eta^{2} \alpha_{2}{ }^{2}}{2 r \mu_{r}(r+\delta)^{2}}+\frac{\lambda_{s}{ }^{2} \eta^{2} \alpha_{1} \alpha_{2}}{r \mu_{s}(r+\delta)^{2}} \\
V^{* * *}\left(N^{* * *}\right)=\frac{\eta}{r+\delta} N^{* * *}+\frac{\varphi}{r}+\frac{\lambda_{m}{ }^{2} \eta^{2}\left(1-\alpha_{1}-\alpha_{2}\right)\left(1+\alpha_{1}+\alpha_{2}\right)}{2 r \mu_{m}(r+\delta)^{2}}+\frac{\lambda_{s}{ }^{2} \eta^{2} \alpha_{1}\left(2-\alpha_{1}\right)}{2 r \mu_{s}(r+\delta)^{2}}+\frac{\lambda_{r}{ }^{2} \eta^{2} \alpha_{2}\left(2-\alpha_{2}\right)}{2 r \mu_{r}(r+\delta)^{2}}
\end{gathered}
$$

where, the optimal innovation level is

$$
N^{* * *}(t)=\frac{\lambda_{m} k_{m}^{* *}+\lambda_{s} k_{s}^{* *}+\lambda_{r} k_{r}^{* *}}{\delta}+\left(N_{0}-\frac{\lambda_{m} k_{m}^{* *}+\lambda_{s} k_{s}^{* *}+\lambda_{r} k_{r}^{* *}}{\delta}\right) e^{-\delta t} .
$$

Prove: same as the proof of proposition 2, omitted.

\subsection{Comparative analysis}

Conclusion 1. In the case of decentralized decision-making mode, the knowledge input of the manufacturer is the same under the two game scenarios; compared with the Nash non- cooperative game, in the Stackelberg master-slave game, the knowledge input amounts of both the supplier and the seller are improved, and the increase ratio is equal to the proportion of their respective knowledge input costs that are shared.

Prove: For known $0<\alpha_{1} \leq \frac{2}{3}\left(1-\alpha_{2}\right)$ and $0<\alpha_{2} \leq$ $\frac{2}{3}\left(1-\alpha_{1}\right)$, from Proposition 1 and Proposition 3 we can know that:

$$
k_{m}^{*}-k_{m}^{* *}=0
$$




$$
\begin{aligned}
k_{s}^{*}-k_{s}^{* *} & =\frac{\lambda_{s} \eta\left(2-\alpha_{1}-2 \alpha_{2}\right)}{2 \mu_{s}(r+\delta)}-\frac{\lambda_{s} \eta \alpha_{1}}{\mu_{s}(r+\delta)}=\frac{\lambda_{s} \eta\left(2-3 \alpha_{1}-2 \alpha_{2}\right)}{2 \mu_{s}(r+\delta)} \\
& =\frac{\lambda_{s} \eta\left(2-\alpha_{1}-2 \alpha_{2}\right)}{2 \mu_{s}(r+\delta)} \cdot \frac{2-3 \alpha_{1}-2 \alpha_{2}}{2-\alpha_{1}-2 \alpha_{2}}=k_{s}^{*} \cdot \beta_{1}^{*}>0 \\
k_{r}^{*}-k_{r}^{* *} & =\frac{\lambda_{r} \eta\left(2-2 \alpha_{1}-\alpha_{2}\right)}{2 \mu_{r}(r+\delta)}-\frac{\lambda_{r} \eta \alpha_{2}}{\mu_{r}(r+\delta)}=\frac{\lambda_{r} \eta\left(2-2 \alpha_{1}-3 \alpha_{2}\right)}{2 \mu_{r}(r+\delta)} \\
& =\frac{\lambda_{r} \eta\left(2-2 \alpha_{1}-\alpha_{2}\right)}{2 \mu_{r}(r+\delta)} \cdot \frac{2-2 \alpha_{1}-3 \alpha_{2}}{2-2 \alpha_{1}-\alpha_{2}}=k_{r}^{*} \cdot \beta_{2}^{*}>0
\end{aligned}
$$

According to Conclusion 1 we can know that, if the manufacturer is the leader of the product collaborative innovation project, when carrying out the collaborative innovation project, it should select the strategy first, therefore its shared cost of knowledge input for the supplier and the seller would not influence its degree of effort on the product collaborative innovation project; as an incentive mechanism, cost sharing can effectively increase the knowledge input of the supplier and the seller, and fully mobilize the enthusiasm of both parties.

Conclusion 2. In the decentralized decision-making mode, compared with the Nash non-cooperative game, in the Stackelberg master-slave game, the product innovation level is improved, and the optimal innovation revenues of the manufacturer, the seller, the supplier, and the whole supply chain have all been improved as well.

Prove: from the expression formulas of $N^{*}$ and $N^{* *}$ we can get: $\left(N^{*}-N^{* *}\right)>0$.

When $0<\alpha_{1} \leq \frac{2}{3}\left(1-\alpha_{2}\right)$ and $0<\alpha_{2} \leq \frac{2}{3}\left(1-\alpha_{1}\right)$, then we have:

$$
\begin{aligned}
& V_{m}^{*}\left(N^{*}\right)-V_{m}^{* *}\left(N^{* *}\right)=\frac{\eta\left(1-\alpha_{1}-\alpha_{2}\right)}{r+\delta}\left(N^{*}-N^{* *}\right) \\
& +\frac{\lambda_{s}^{2} \eta^{2}\left(2-3 \alpha_{1}-2 \alpha_{2}\right)^{2}}{8 r \mu_{s}(r+\delta)^{2}}+\frac{\lambda_{r}{ }^{2} \eta^{2}\left(2-2 \alpha_{1}-3 \alpha_{2}\right)^{2}}{8 r \mu_{r}(r+\delta)^{2}}>0 \\
& V_{s}^{*}\left(N^{*}\right)-V_{s}^{* *}\left(N^{* *}\right)=\frac{\eta \alpha_{1}}{r+\delta}\left(N^{*}-N^{* *}\right) \\
& +\frac{\lambda_{s}{ }^{2} \eta^{2} \alpha_{1}\left(2-3 \alpha_{1}-2 \alpha_{2}\right)}{4 r \mu_{s}(r+\delta)^{2}}+\frac{\lambda_{r}{ }^{2} \eta^{2} \alpha_{1}\left(2-2 \alpha_{1}-3 \alpha_{2}\right)}{2 r \mu_{r}(r+\delta)^{2}}>0 \\
& V_{r}^{*}\left(N^{*}\right)-V_{r}^{* *}\left(N^{* *}\right)=\frac{\eta \alpha_{2}}{r+\delta}\left(N^{*}-N^{* *}\right) \\
& +\frac{\lambda_{r}{ }^{2} \eta^{2} \alpha_{2}\left(2-2 \alpha_{1}-3 \alpha_{2}\right)}{4 r \mu_{r}(r+\delta)^{2}}+\frac{\lambda_{s}{ }^{2} \eta^{2} \alpha_{2}\left(2-3 \alpha_{1}-2 \alpha_{2}\right)}{2 r \mu_{s}(r+\delta)^{2}}>0
\end{aligned}
$$

In summary, $V^{*}\left(N^{*}\right)-V^{* *}\left(N^{* *}\right)>0$. The conclusion is proved.

It can be seen from conclusion 2 that in the product collaborative innovation project led by the manufacturer, by implementing the know input cost-sharing strategy, the Pareto improvement of the member companies participating in the project and the whole supply chain could be realized, forming a win-win situation, therefore, compared with the Nash noncooperative game, member companies in the project-based supply chain are more inclined to choose the Stackelberg master-slave game, that is, the collaborative innovation project to be carried out needs to have a leader company or a core enterprise.

\section{CENTRALIZED DECISION-MAKING MODE}

\subsection{Cooperative game}

It is assumed that in a project-based supply chain, the manufacturer, the supplier and the seller constitute a unified interest group, that is, they take the revenue maximization of the whole supply chain as the common goal to negotiate and determine their respective optimal knowledge input amount, so as to improve the product innovation level to the greatest extent. At the same time, the cost sharing of knowledge input belongs only to the transfer of funds within the supply chain, so the cost-sharing ratio can be any value within $[0,1]$, and it will not affect the strategic choice of each participating company. The objective function of the supply chain system is:

$$
J=\max _{k_{M}, k_{S}, k_{R}} \int_{0}^{\infty} e^{-r t}\left[(\varphi+\eta N(t))-\frac{\mu_{M}}{2} k_{M}{ }^{2}-\frac{\mu_{S}}{2} k_{S}{ }^{2}-\frac{\mu_{R}}{2} k_{R}{ }^{2}\right] \mathrm{d} t
$$

Through solving, we can obtain Propositions 5 and 6 as follows, the proof process is similar to Section 3 and is omitted.

Proposition 5. In the case of cooperative games, the optimal knowledge input amounts of the manufacturer, the supplier and the seller in the three-stage project-based supply chain are:

$$
\begin{aligned}
& k_{m}^{* * * *}=\frac{\lambda_{m} \eta}{\mu_{m}(r+\delta)} \\
& k_{s}^{* * * *}=\frac{\lambda_{s} \eta}{\mu_{s}(r+\delta)} \\
& k_{r}^{* * * *}=\frac{\lambda_{r} \eta}{\mu_{r}(r+\delta)}
\end{aligned}
$$

Proposition 6. In the case of cooperative games, the optimal innovation revenue of the three-stage project-based supply chain is:

$$
\begin{aligned}
& V^{* * * *}\left(N^{* * * *}\right)=\frac{\eta}{r+\delta} N^{* * * *}+\frac{\varphi}{r}+\frac{\lambda_{m}{ }^{2} \eta^{2}}{2 r \mu_{m}(r+\delta)^{2}} \\
& +\frac{\lambda_{s}^{2} \eta^{2}}{2 r \mu_{s}(r+\delta)^{2}}+\frac{\lambda_{r}{ }^{2} \eta^{2}}{2 r \mu_{r}(r+\delta)^{2}},
\end{aligned}
$$

Wherein, the optimal innovation level is:

$$
\begin{aligned}
& N^{* * * *}(t)=\frac{\lambda_{m} k_{m}^{* * * *}+\lambda_{s} k_{s}^{* * * *}+\lambda_{r} k_{r}^{* * * *}}{\delta} \\
& +\left(N_{0}-\frac{\lambda_{m} k_{m}^{* * * *}+\lambda_{s} k_{s}^{* * * *}+\lambda_{r} k_{r}^{* * * *}}{\delta}\right) e^{-\delta t} .
\end{aligned}
$$

\subsection{Comparative analysis}

Conclusion 3. In the centralized decision-making mode, the optimal knowledge input amounts of the manufacturer, the supplier, and the seller are all higher than that in the decentralized decision-making mode.

Proof: When there are $0<\alpha_{1} \leq \frac{2}{3}\left(1-\alpha_{2}\right)$ and $0<\alpha_{2} \leq$ $\frac{2}{3}\left(1-\alpha_{1}\right)$, from Propositions 1 and 5 we can know that: 


$$
\begin{aligned}
& k_{m}^{* * *}-k_{m}^{*}=\frac{\lambda_{m} \eta}{\mu_{m}(r+\delta)}-\frac{\lambda_{m} \eta\left(1-\alpha_{1}-\alpha_{2}\right)}{\mu_{m}(r+\delta)} \\
& =\frac{\lambda_{m} \eta\left(\alpha_{1}+\alpha_{2}\right)}{\mu_{m}(r+\delta)}>0, \\
& k_{s}^{* * * *}-k_{s}^{*}=\frac{\lambda_{s} \eta}{\mu_{s}(r+\delta)}-\frac{\lambda_{s} \eta\left(2-\alpha_{1}-2 \alpha_{2}\right)}{2 \mu_{s}(r+\delta)} \\
& =\frac{\lambda_{s} \eta\left(\alpha_{1}+2 \alpha_{2}\right)}{2 \mu_{s}(r+\delta)}>0, \\
& k_{r}^{* * *}-k_{r}^{*}=\frac{\lambda_{r} \eta}{\mu_{r}(r+\delta)}-\frac{\lambda_{r} \eta\left(2-2 \alpha_{1}-\alpha_{2}\right)}{2 \mu_{r}(r+\delta)} \\
& =\frac{\lambda_{r} \eta\left(2 \alpha_{1}+\alpha_{2}\right)}{2 \mu_{r}(r+\delta)}>0 .
\end{aligned}
$$

From conclusion 3 we can know that, in the case of centralized decision-making mode, the member companies participating in the product collaborative innovation project will take the entire supply chain as the decision-making goal, thus they could effectively coordinate the knowledge input behaviors among the member companies in the project-based supply chain, so that the knowledge input of each participating company is maximized, thereby jointly promoting the successful completion of the collaborative innovation project.

Conclusion 4. In the centralized decision-making mode, the product innovation level and the overall innovation revenue of the three-stage supply chain are both higher than that in the decentralized decision-making mode.

Proof: from the expression formulas of $N^{*}$ and $N^{* * *}$ we can easily get $\left(N^{* * *}-N^{*}\right)>0$. When $0<\alpha_{1} \leq \frac{2}{3}\left(1-\alpha_{2}\right)$ and $0<\alpha_{2} \leq \frac{2}{3}\left(1-\alpha_{1}\right)$, we have:

$$
\begin{aligned}
& V\left(N^{* * *}\right)-V^{*}\left(N^{*}\right)=\frac{\eta}{r+\delta}\left(N^{* * * *}-N^{*}\right)+ \\
& \frac{\lambda_{m}{ }^{2} \eta^{2}\left(\alpha_{1}+\alpha_{2}\right)^{2}}{2 r \mu_{m}(r+\delta)^{2}}+\frac{\lambda_{s}{ }^{2} \eta^{2}\left(\alpha_{1}+2 \alpha_{2}\right)^{2}}{2 r \mu_{s}(r+\delta)^{2}} \\
& +\frac{\lambda_{r}{ }^{2} \eta^{2}\left(2 \alpha_{1}+\alpha_{2}\right)^{2}}{2 r \mu_{r}(r+\delta)^{2}}>0 .
\end{aligned}
$$

According to conclusion 4 we can know that, in the centralized decision-making mode, member companies in the supply chain will no longer be limited to their own revenues, but will make their decisions with the revenue maximization of the whole supply chain as the goal, thereby achieving the improvement of product innovation level and the revenue increment of the whole supply chain, thereby realizing the Pareto's optimality.

\section{NUMERICAL EXAMPLES}

Assume that the parameters in the model are set as follows: $\mu_{m}=0.5, \mu_{s}=0.4, \mu_{r}=0.2, \lambda_{m}=0.4, \lambda_{s}=0.3, \lambda_{r}=0.1$, $\delta=0.1, \varphi=0.5, \eta=0.4, \quad r=0.1, N(0)=N_{0}=3$, $\alpha_{1}=0.24, \alpha_{2}=0.16$. From formulas (6), (7), and (8) we can know that, $k_{m}^{* *}=0.96, k_{s}^{* *}=0.36, k_{r}^{* *}=0.16$, from formulas (37), (38), and (39) we can know $k_{m}^{* * * *}=1.6, k_{s}^{* * * *}=1.5, k_{r}^{* * * *}=1$.
From formulas (9) and (10) we can know $\beta_{1}^{*}=2 / 3$ and $\beta_{2}^{*}=0.98 \quad, \quad$ obviously, there are $k_{m}^{*}=k_{m}^{* *}<k_{m}^{* * *}$, $k_{s}^{* *}<k_{s}^{*}<k_{s}^{* * *}$ and $k_{r}^{* *}<k_{r}^{*}<k_{r}^{* * * *}$, and they satisfy $k_{s}^{*}-k_{s}^{* *}=0.72=k_{S}^{*} \cdot \beta_{1}^{*}$ and $k_{r}^{*}-k_{r}^{* *}=0.52=k_{r}^{*} \cdot \beta_{2}^{*}$. The above results are consistent with Conclusions 1 and 3 . Furthermore, through computer simulation, we can get the change trends of the optimal innovation revenues of the manufacturer, the supplier, and the seller with time under the decentralized decision-making mode, and the change trajectories of the overall innovation revenue and the innovation level of the supply chain under the three game scenarios, as shown in Figures 2-3.

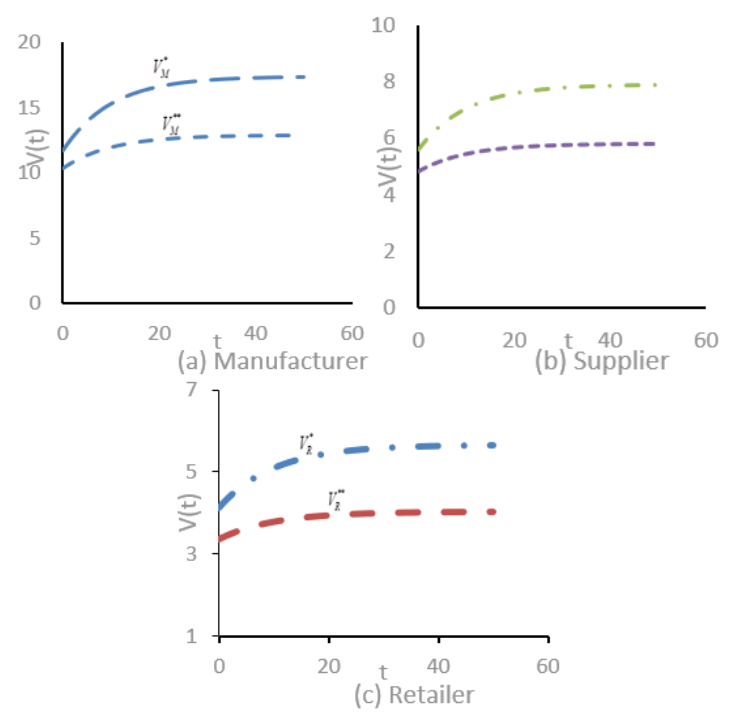

Figure 2. Comparative analysis of the optimal innovation revenues of different subjects under the decentralized decision-making mode
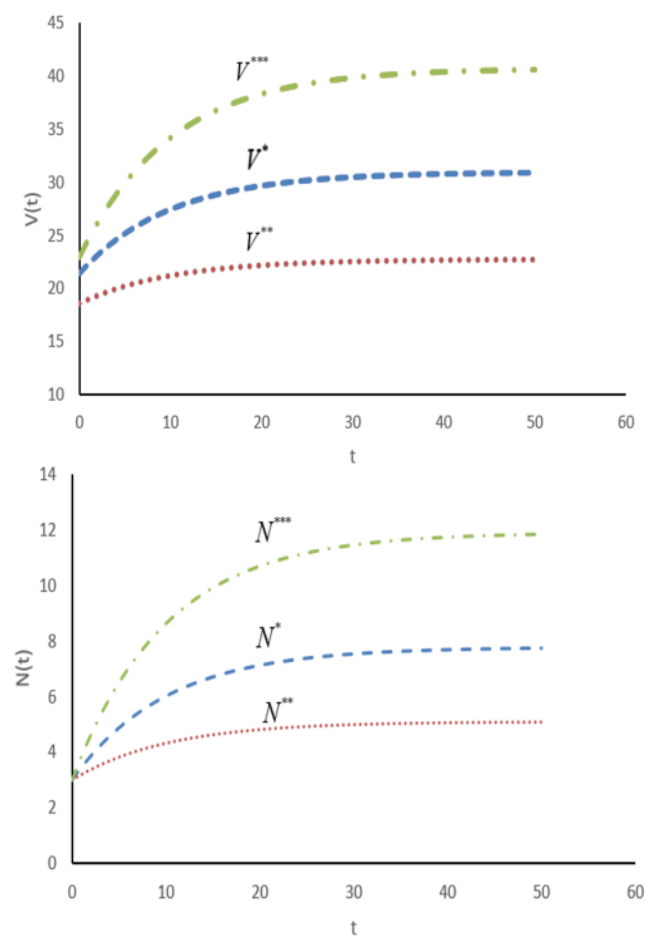

Figure 3. Comparative analysis of the overall optimal innovation revenues of the supply chain and optimal product innovation level under three scenarios 
From Figures 2 and 3 we can know the optimal innovation revenues of the manufacturer, the supplier, and the seller in the decentralized decision-making mode under the three game scenarios, the overall innovation revenue level of the supply chain has a time-stable trend, and it increases with the time, the growth rate in the early period was faster, then in later period it gradually became flat and eventually converged to equilibrium, indicating that the dynamic change of the supply chain system has been effectively regulated. In the case of Stackelberg master-slave game, the optimal innovation revenue level of the manufacturer, the supplier, the seller, and the overall innovation revenue level of the supply chain were always higher than those under the Nash non-cooperative game scenario. In the centralized decision-making mode, the overall innovation revenue of the supply chain reached the highest, which was better than that in the decentralized decision-making mode, and this is consistent with Conclusions 2 and 4 . From Figure 3 we can know that, although the optimal innovation revenue of the supply chain increases with time, the increase speed becomes slower and slower, indicating that the innovation level shows a marginal decreasing trend as the supply chain members increase their knowledge input.

\section{CONCLUSION}

Considering the effect of enterprise knowledge input on the collaborative innovation project in the three-stage projectbased supply chain (consisting of a single product manufacturer, a single component supplier and a single product seller), this paper constructed a differential time model based on continuous time, and successively investigated the optimal knowledge input of member enterprises, optimal innovation revenue, optimal product innovation level and the overall revenue of the supply chain system under three scenarios: the cooperative game (centralized decision-making mode), the Stackelberg master-slave game and the Nash noncooperative game (decentralized decision-making mode). Through the comparative analysis and numerical simulation of the model results, the following conclusions can be drawn: under the centralized decision-making mode, the knowledge input and collaborative innovation of enterprises were optimized, leading to the maximal overall revenue of the supply chain. Under the two decentralized decision-making modes, the manufacturer had the same knowledge input; however, the supplier and the seller both had higher knowledge input amount, enterprise revenue and collaborative innovation level under the Stackelberg master-slave game than under the Nash non-cooperative game; the increase ratios of knowledge input of the supplier and the seller are equal to the proportions of their respective knowledge input costs that are shared.

\section{ACKNOWLEDGMENTS}

The authors acknowledge funding from the National Social Science Foundation of China (Project No. 18CGL003) and Research Center for Enterprise Management in Chongqing Technology and Business University, as well as the contributions from all partners of the mentioned project. Besides, Hao Yifei is the corresponding author and can be contacted at: haoyf@ctbu.edu.cn.

\section{REFERENCES}

[1] Donselaar, K.V., Kopczak, L.R., Wouters, M. (2001). The use of advance demand information in a projectbased supply chain. European Journal of Operational Research, $\quad 130(3)$ : 519-538. https://doi.org/10.1016/s0377-2217(99)00411-7

[2] Estrada, I., Faems, D., Faria, P.D. (2016). Coopetition and product innovation performance: The role of internal knowledge sharing mechanisms and formal knowledge protection mechanisms. Industrial Marketing Management, 53(2): 56-65. https://doi.org/10.1016/j.indmarman.2015.11.013

[3] Han, W., Yuan, H., Macbeth, D. (2017). Performance measurement of cross-culture supply chain partnership: A case study in the Chinese automotive industry. International Journal of Production Research, 56(9): 115. https://doi.org/10.1080/00207543.2017.1377357

[4] Chen, Y.Z., Chen, W. (2019). Incentive contracts of knowledge investment for cooperative innovation in project-based supply chain with double moral hazard. Soft Computing, 23(1): 1-10. https://doi.org/10.1007/s00500-019-03894-8

[5] Wu, G.D. (2013). Knowledge collaborative incentive based on inter-organizational cooperative innovation of project-based supply chain. Journal of Industrial Engineering and Management, 6(4): 1065-1081. https://doi.org/10.3926/jiem.772

[6] Saunders, L.W., Kleiner, B.M., Mccoy, A.P., Lingard, H. Mills, T., Blismas, N., Wakefield, R. (2015). The effect of early supplier engagement on social sustainability outcomes in project-based supply chains. Journal of Purchasing \& Supply Management, 21(4): 285-295. https://doi.org/10.1016/j.pursup.2015.05.004

[7] Nasr, E.S., Kilgour, M.D., Noori, H. (2015). Strategizing niceness in co-opetition: The case of knowledge exchange in supply chain innovation projects. European Journal of Operational Research, 244(3): 845-854. https://doi.org/10.1016/j.ejor.2015.02.011

[8] Lai, X.D., Wu, G.D., Shi, J.G. (2015). Project valueadding optimization of project-based supply chain under dynamic reputation incentives. International Journal of Simulation Modelling, 14(1): 121-133. https://doi.org/10.2507/ijsimm14(1)co1

[9] Wu, G.D., Tang, D.Z. (2015). Inter-organizational cooperative innovation of project-based supply chains under consideration of monitoring signals. International Journal of Simulation Modelling, 14(3): 539-550. https://doi.org/10.2507/IJSIMM14(3)CO14

[10] Preeker, T., Giovanni, D.P. (2018). Coordinating innovation projects with high tech suppliers through contracts. Research Policy, 47(6): 1161-1172. https://doi.org/10.1016/j.respol.2018.04.003

[11] Chen, W., Yang, B., Lin, C. (2016). Partner selection mechanism of cooperative innovation in project-based supply chain based on the effect of knowledge level. Advances in Modelling and Analysis B, 59(1): 146-163.

[12] Yan, T., Azadegan, A. (2017). Comparing interorganizational new product development strategies: Buy or ally; supply-chain or non-supply-chain partners. International Journal of Production Economics, 183(1): 21-38. https://doi.org/10.1016/j.ijpe.2016.09.023

[13] Min, L., Wang, Z., Zhao, X. (2018). The role of indigenous technological capability and interpersonal 
trust in supply chain learning. Industrial Management \& Data Systems, 118(6): 1052-1070. https://doi.org/10.1108/IMDS-08-2017-0350

[14] Ajmal, M.M., Koskinen, K.U. (2008). Knowledge transfer in project-based organizations: An organizational culture perspective. Project Management Journal, 39(1): 7-15. https://doi.org/10.1002/pmj.20031

[15] David, T.R., Lakemond, N., Melander, L. (2017). Integrating supplier knowledge in new product development projects: Decoupled and coupled approaches. Journal of Knowledge Management, 21(5): 1035-1052. https://doi.org/10.1108/JKM-10-2016-0438

[16] Song, H., Shao, Y.F., Dai, Y. (2017). Contract selection under moral hazard and capital restrictions of service providers in R\&D outsourcing: Punishments or rewards. Science Research Management, 38(12): 122-133. https://doi.org/10.CNKI:SUN:KYGL.0.2017-12-014 